\title{
ON THE LINE GRAPH OF A SYMMETRIC BALANCED INCOMPLETE BLOCK DESIGN
}

\author{
BY \\ A. J. HOFFMAN $\left({ }^{1}\right)$ AND D. K. RAY-CHAUDHURI
}

1. Introduction. We shall study the relations between an infinite family of finite graphs and the eigenvalues of the corresponding adjacency matrices. All graphs we consider are undirected, finite, with at most one edge joining a pair of vertices, and with no edge joining a vertex to itself. Also, they are all connected and regular (every vertex has the same valence). If $G$ is a graph, its adjacency matrix $A=A(G)$ is given by

$$
a_{i j}=\left\{\begin{array}{l}
1 \text { if } i \text { and } j \text { are adjacent vertices, } \\
0 \text { otherwise. }
\end{array}\right.
$$

The line graph $L(G)$ (also called the interchange graph, and the adjoint graph) of a graph $G$ is the graph whose vertices are the edges of $G$. With two vertices of $L(G)$ adjacent if and only if the corresponding edges of $G$ are adjacent.

There have been several investigations in recent years of the extent to which a regular connected graph is characterized by the eigenvalues of its adjacency matrix, especially in the case of line graphs (see [4] for a bibliography, and [2]). Most germane to the present investigation is the result of [4], which we now briefly describe.

Let $\Pi$ be a finite projective plane with $n+1$ points on a line. We regard $\Pi$ as a bipartite graph with $2\left(n^{2}+n+1\right)$ vertices, which are all points and lines of $\Pi$, with two vertices adjacent if and only if one is a point, the other is a line, and the point is on the line. Let $L(\Pi)$ be the line graph of $\Pi$. A useful way of visualizing $L(\Pi)$ is to imagine its vertices as the 1's in the incidence matrix of $\Pi$ (see [4]), with two 1's corresponding to adjacent vertices if and only if they are in the same row or column of the incidence matrix. Then $L(\Pi)$ is a regular connected graph with $(n+1)$ $\cdot\left(n^{2}+n+1\right)$ vertices whose adjacency matrix has

$$
2 n,-2, n-1 \pm \sqrt{ } n
$$

as its distinct eigenvalues. It is shown in [4] that any regular connected graph on $(n+1)\left(n^{2}+n+1\right)$ vertices whose distinct eigenvalues are given by (1.1) must be isomorphic to the line graph of a plane $\Pi$ with $n+1$ points on a line. (It is, of course, impossible for (1.1) to distinguish

Received by the editors May 13, 1964.

$\left({ }^{1}\right)$ This research was supported in part by the Office of Naval Research under Contract No. Nonr 3775(00), NR 047070. 
between nonisomorphic planes of the same order $n$.)

In this paper we generalize this result to symmetric balanced incomplete block designs (also called $\lambda$-planes). An $\operatorname{SBIB} \Pi(v, k, \lambda)$ can be conceived as a bipartite graph on $v+v$ vertices, each vertex having valence $k$, with any two vertices in the same part adjacent to exactly $\lambda$ vertices of the other part. It is assumed that $0<\lambda<k<v$, and it is well known that

$$
\lambda=\frac{k(k-1)}{v-1} .
$$

Just as in [4], one readily shows (see $\$ 4$ ) that $L(\Pi)$ is a regular connected graph on $v k$ vertices, and its adjacency matrix has

$$
2 k-2,-2, k-2 \pm \sqrt{ }(k-\lambda)
$$

as its distinct eigenvalues. We then raise the question: if $H$ is a regular connected graph on $v k$ vertices, with (1.3) as the distinct eigenvalues of its adjacency matrix, is $H$ isomorphic to some $L(\Pi(v, k, \lambda))$ ?

The answer is yes, unless $v=4, k=3, \lambda=2$, in which case there is exactly one exception.

2. Outline of proof. A (three-fingered) claw is a graph consisting of four vertices $0,1,2,3$ such that 0 is adjacent to $1,2,3$ but $i$ is not adjacent to $j(i, j=1,2,3)$. We shall denote such a claw by the notation $(0 ; 1,2,3)$. It is clear that a line graph contains no claw, and, conversely, if we can show under suitable hypotheses that $H$ contains no claw, then the remainder of the proof that $H \cong L(\Pi)$ will be quite straightforward. Our central problem then is to prove $H$ contains no claw.

Let $A=A(H)$, and consider the matrix

$$
B=A^{2}-(2 k-2) I-(k-2) A .
$$

We shall show below in $\$ 4$ that, for each $i$,

$$
\sum_{j} b_{i j}\left(b_{i j}-1\right)=2(\lambda-1)(k-1) .
$$

Consider also

$$
C=A^{2}-(2 k-2) I-(k-2) A-(J-I-A),
$$

where $J$ is a matrix of all 1 's.

We shall show in $\$ 4$ that, for each $i$,

$$
\sum_{j} c_{i j}\left(c_{i j}-1\right)=2(v-k)(k-\lambda) .
$$

After further preliminaries, we consider the case when we assume that $H$ is edge regular (i.e., every edge is contained in the same number of triangles). With this additional hypothesis, the nonexistence of claws is 
readily established, the only case requiring any effort being $k=4$. Next, we consider the case when $H$ is not edge regular. Then claws must exist satisfying certain properties. But we show that, apart from the exception cited in the introduction, these claws would produce violations of (2.2) or (2.4). These violations are the result of a counting process, and the counting is facilitated by showing that certain graphs canhot be subgraphs of $H$. (The discussion of the edge regular case also uses the nonexistence of some subgraphs.) A list of the "impossible" subgraphs is given in $\$ 3$, and we now explain the principles used in proving these subgraphs impossible. They are based on elementary facts about eigenvalues and eigenvectors of symmetric matrices.

The first principle is: if $K$ is a subgraph of $H$, if $M=M(K)$ is the adjacency matrix of $K$, if -2 is an eigenvalue of $M$, and $x$ the corresponding eigenvector, then the sum of the coordinates of $x$ must be zero.

The reason is as follows. Let $y$ be the row vector with $v k$ components obtained by adjoining to the vector $x$ additional coordinates all zero. It easily follows that $y A y^{T}=-2 y y^{T}$. Since -2 is the minimum eigenvalue of $A, y$ is an eigenvector of $A$ corresponding to the eigenvalue -2 . Now $2 k-2$ is also an eigenvalue of $A$, corresponding to the eigenvector $(1,1, \ldots, 1)$ (see [3] for a brief justification). In a symmetric matrix, two eigenvectors corresponding to different eigenvalues must be orthogonal. Hence, $y$ must be orthogonal to $(1,1, \cdots, 1)$, i.e., the sum of the coordinates of $x$ is 0 .

Thus, the graph

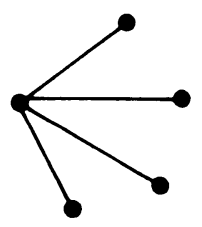

whose corresponding adjacency matrix is

$$
\left(\begin{array}{lllll}
0 & 1 & 1 & 1 & 1 \\
1 & 0 & 0 & 0 & 0 \\
1 & 0 & 0 & 0 & 0 \\
1 & 0 & 0 & 0 & 0 \\
1 & 0 & 0 & 0 & 0
\end{array}\right),
$$

cannot be a subgraph of $H$, since $(-2,1,1,1,1)$ is an eigenvector of (2.5), with -2 the corresponding eigenvalue.

Our second principle is that, if the sum of the coordinates of $x$ is 0 , then, if $a$ is any vertex of $H$ not in $K$, the sum of the coordinates of $x$ corresponding to vertices of $K$ adjacent to $a$ must be 0 . The proof is a direct application of the minimum characterization of the least eigenvalue. 
This principle makes the following graph impossible:

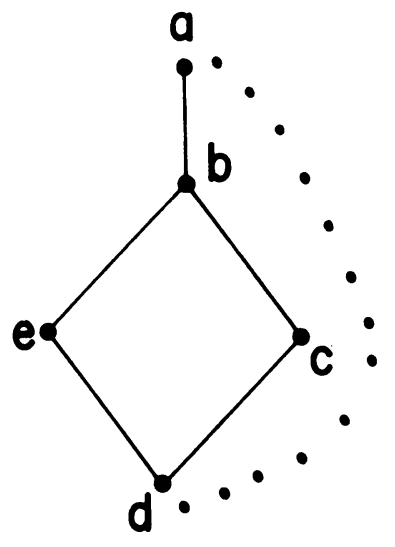

where the dotted line indicates that there may or may not be an edge of $H$. For the graph $K$ whose vertices are $b, c, d, e$ has $(1,-1,1,-1)$ as eigenvector of the corresponding adjacency matrix.

3. Impossible subgraphs. We first list some subgraphs impossible because of the first principle. Accompanying each vertex will be a latin letter (for later reference) and a number giving the coordinate of the corresponding eigenvector.
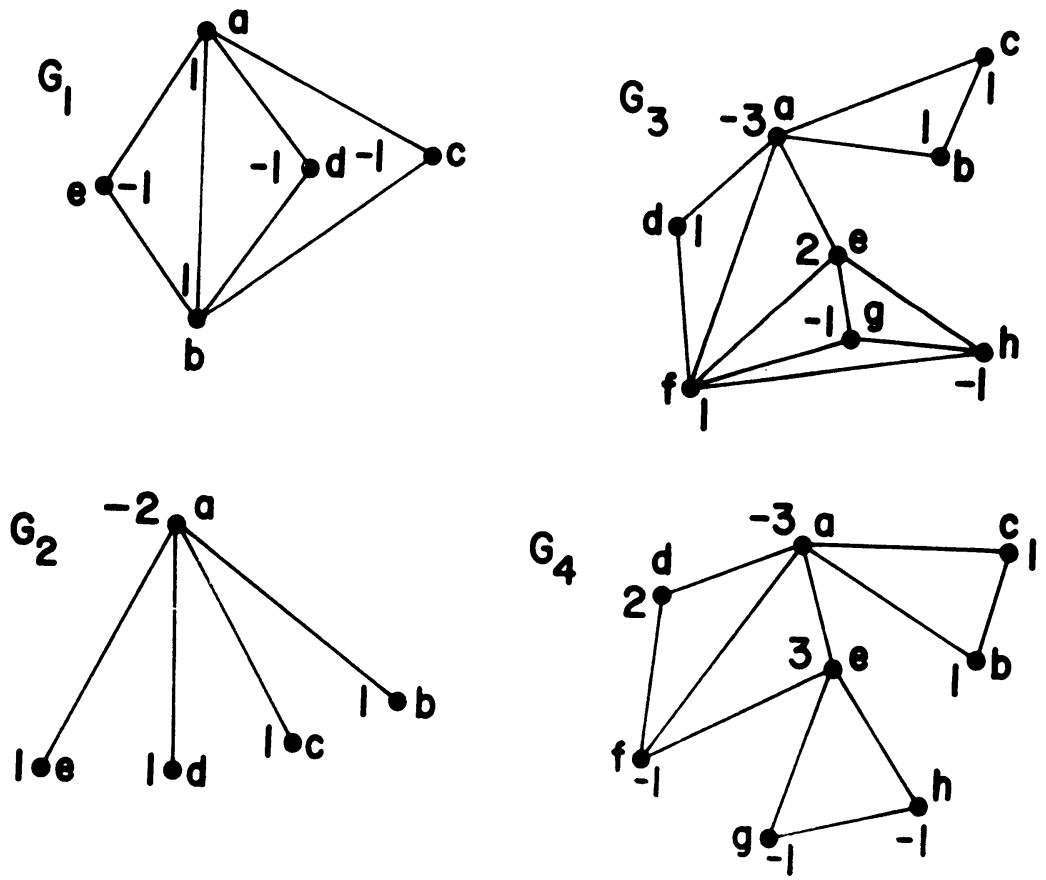

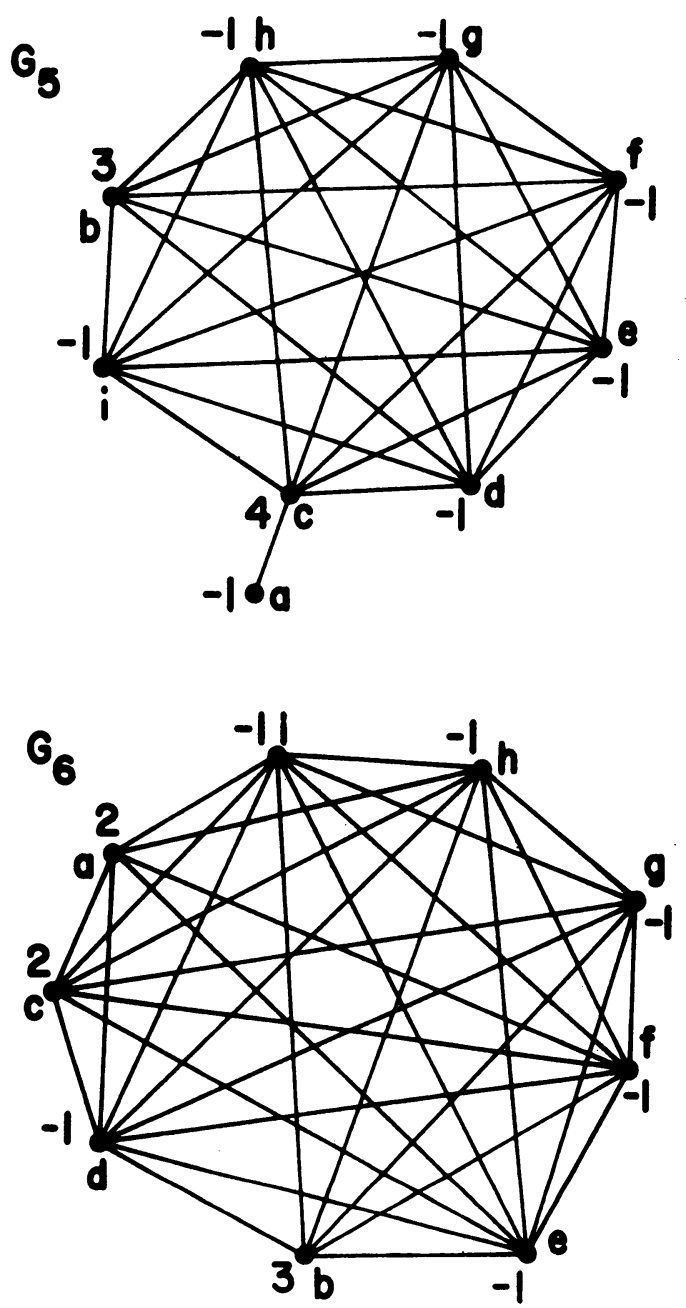

We now list some subgraphs impossible because of the second principle. The "other" vertex is denoted by the letter $\alpha$. A dotted line signifies that the edge may be present or absent.
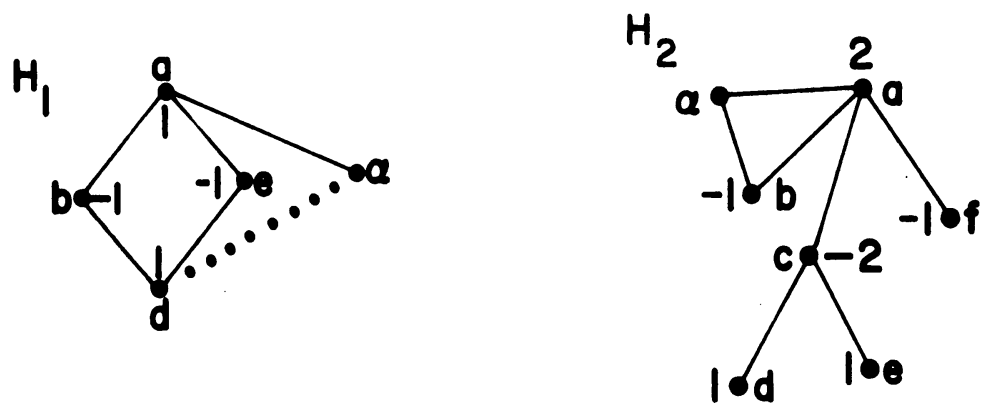

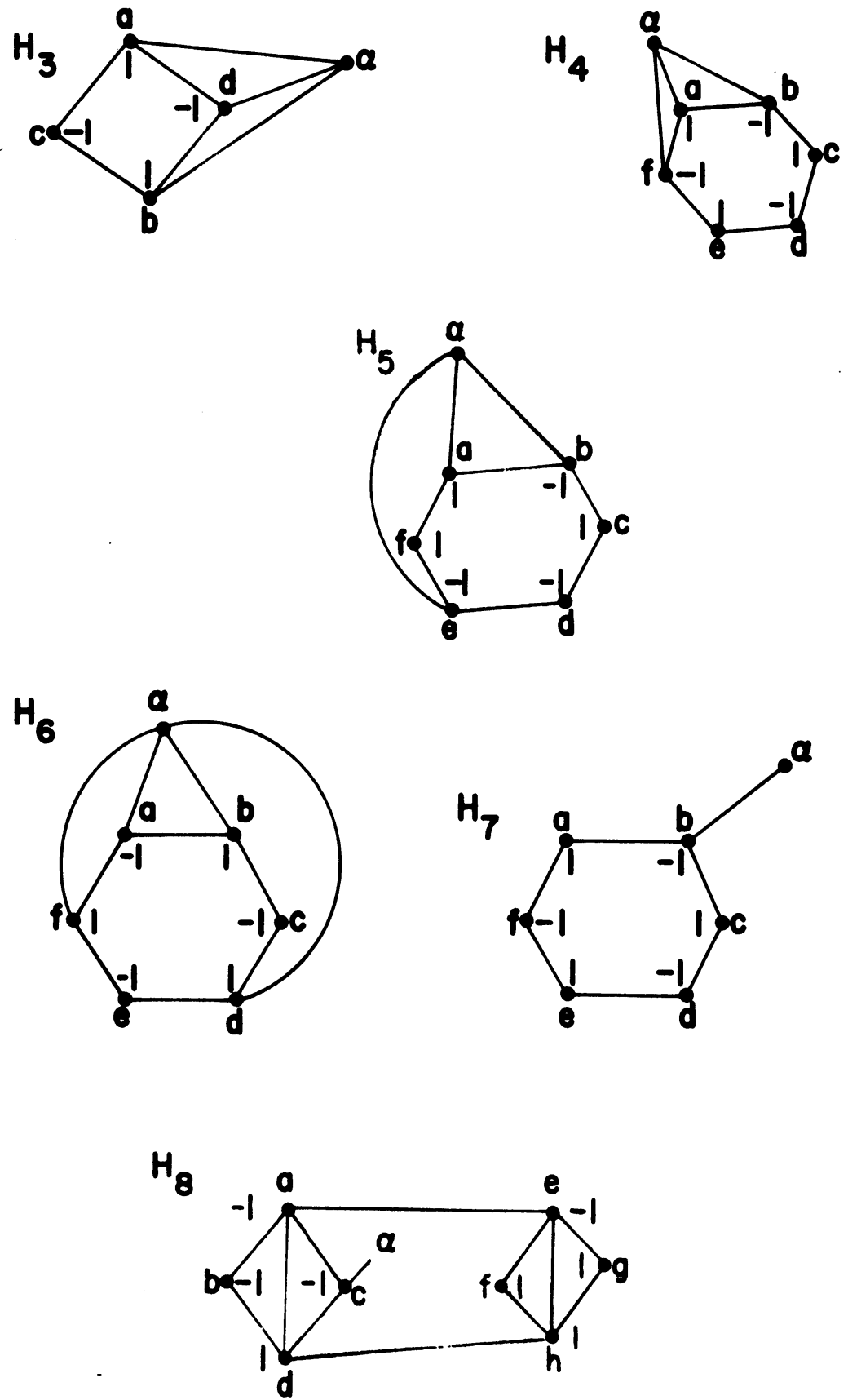

4. Some preliminaries on matrices. We begin with two lemmas.

LEMMA 4.1. Let $G$ be a regular connected graph on $2 v$ vertices, $A=A(G)$. The distinct eigenvalues of $A$ are given by 


$$
k,-k, \sqrt{ }(k-\lambda),-\sqrt{ }(k-\lambda)
$$

if and only if $G$ is $\Pi(v, k, \lambda)$.

Proof. Assume $G \cong \Pi(v, k, \lambda)$. Then $A$ may be written as

$$
A(G)=\left(\begin{array}{ll}
O & B \\
B^{T} & O
\end{array}\right)
$$

where $B$ is a matrix of order $v$, and

$$
B B^{T}=B^{T} B=(k-\lambda) I+\lambda J .
$$

It is well known [3] that this form means that the eigenvalues of $A$ are the numbers whose squares are the eigenvalues of $B B^{T}$. But from (4.3), the eigenvalues of $B B^{T}$ are $k^{2}$ and $k-\lambda$. Also since the multiplicity of the eigenvalue $k$ of the matrix $A$ which is the adjacency matrix of a regular connected graph is $1[3], k^{2}$ is a simple root of $B B^{T}$.

Conversely, if the distinct eigenvalues of $A=A(G)$ are given by (4.1), then $G$ is bipartite [3], hence of the form (4.2). It follows that $B B^{T}$ is a matrix of order $v$, whose distinct eigenvalues are $k^{2}$ and $k-\lambda$. Further, because $G$ is regular, every row and column sum of $B$, hence every row and column sum of $B B^{T}$ is the same. Therefore, if we set $u=(1, \cdots, 1)$, $u$ is an eigenvector of $B B^{T}$, corresponding to the dominant eigenvalue of $B B^{T}$, so we must have $B B^{T} u=k^{2} u$. Further, $J$ commutes with $B B^{T}$. Hence the eigenvalues of $B B^{T}-((k-\lambda) I+\lambda J)$ are all 0 . Therefore, since $B B^{T}$ is symmetric $B B^{T}=(k-\lambda) I+\lambda J$, which was to be proven.

Lemma 4.2. Let $H=L(\Pi(v, k, \lambda))$. Then the distinct eigenvalues of $A(H)$ are given by

$$
2 k-2,-2, k-2+\sqrt{ }(k-\lambda), k-2-\sqrt{ }(k-\lambda) .
$$

Conversely, let $H$ be a graph with vk vertices, and known to be the line graph of a regular connected graph with $2 v$ vertices. If the distinct eigenvalues of $A(H)$ are given by (4.4), then $H \cong L(\Pi(v, k, \lambda))$.

Proof. Assume $H=L(\Pi(v, k, \lambda))$. Let $K$ be the matrix with $2 v$ rows (the first $v$ rows corresponding to one part of $\Pi(v, k, \lambda)$, the remaining rows corresponding to the other part of $\Pi(v, k, \lambda)$, and $v k$ columns corresponding to the edges of $\Pi(v, k, \lambda)$. An entry in $K$ is 1 if the corresponding vertex and edge are incident, 0 otherwise. Then

$$
K K^{T}=k I+A(G),
$$

where $A(G)$ is as in (4.2).

$$
K^{T} K=2 I+A(H) .
$$

The nonzero eigenvalues of $K K^{T}$ and $K^{T} K$ are the same. Further, 0 is an eigenvalue of $K^{T} K$, since $K$ has more columns than rows, and 0 is an 
eigenvalue of $K K^{T}$ since the sum of the first $v$ rows of $K$ minus the sum of the last $v$ rows is 0 . Hence $K K^{T}$ and $K^{T} K$ have exactly the same set of distinct eigenvalues. From (4.5), (4.6) and (4.1), we infer (4.4).

Conversely, if $H$ is the line graph with $v k$ vertices, if $H=L(G)$, where $G$ has $2 v$ vertices, and if the distinct eigenvalues $A(H)$ are given by (4.4), then the above discussion is completely reversible unless the rows of $K$ are not linearly dependent, (i.e., $G$ is not bipartite). This would mean that $A(G)$ has for its distinct eigenvalues $k, \sqrt{ }(k-\lambda)$ and $-\sqrt{ }(k-\lambda)$. Then the polynomial of $G$ (see [3]) would be

$$
\frac{2}{\lambda}\left(x^{2}-(k-\lambda)\right)
$$

so

$$
(A(G))^{2}-(k-\lambda) I=\frac{\lambda}{2} J .
$$

In other words, the diagonal element of $(A(G))^{2}$ would be $k-\lambda+\lambda / 2$. But since $G$ is regular and $k$ is the dominant eigenvalue of $A(G)$, every row sum of $A(G)$ must be $k$. Therefore, every diagonal element of $(A(G))^{2}$ must be $k$. This is a contradiction.

Henceforth, we assume $H$ is a regular connected graph with $v k$ vertices, and $A(H)$ has (4.4) as its distinct eigenvalues. We also write $A=A(H)$.

Lemma 4.3. The matrix $A$ satisfies the equation

(4.7) $(A+2 I)(A-(k-2+\sqrt{ }(k-\lambda)) I)(A-(k-2-\sqrt{ }(k-\lambda)) I)=2 \lambda J$.

Proof. See [3].

Lemma 4.4. If $B$ is defined by (2.1), then (2.2) holds. If $C$ is defined by (2.3), then (2.4) holds.

Proof. It is clear that (2.2) and (2.4) can be established if we can calculate the diagonal element of any power of $A$, and of any power of $A$ multiplied by $J$. Since the row sums of $A$ are all $(2 k-2)$, it follows that $A^{t} J=(2 k-2)^{t} J$. Also, we know the diagonal entries of $I, A$, and $A^{2}$. Since the left side of (4.7) is a third degree polynomial in $A$, we can calculate the diagonal entires of $A^{3}$. Multiplying (4.7) by $A$, we can then calculate the diagonal elements of $A^{4}$.

5. Some preliminaries on claws. In this section, we assume a claw $0,1,2,3$, in the form

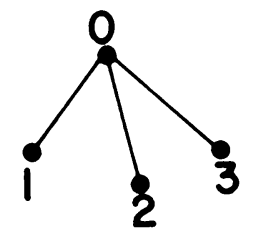


The subgraph of $H$ determined by the vertices $x_{1}, \cdots, x_{n}$ will be written as $H\left(x_{1}, \cdots, x_{n}\right)$. We define

$$
\begin{aligned}
& S_{i}=\{x \mid x \text { is adjacent to } 0 \text { and } i \text {, but not to } j \text { or } k\} . \\
& S_{i j}=\{x \mid x \text { is adjacent to } 0, i \text { and } j, \text { but not to } k\} .
\end{aligned}
$$

Note that no vertex is adjacent to $0,1,2,3$. If a vertex (say 4) is adjacent to $0,1,2,3$, then $H(0,1,2,3,4)=G_{1}(a, c, d, e, b)$. (The equality $H(0,1,2,3,4)=G_{1}(a, c, d, e, b)$ means that the graph $G_{1}$ is the same as the graph $H(0,1,2,3,4)$ and the vertices $(0,1,2,3,4)$ are respectively identified with the vertices $(a, c, d, e, b)$.) Also, every vertex other than $1,2,3$ adjacent to 0 must be in some $S_{i}$ or some $S_{i j}$. Otherwise, we would have graph $G_{2}$. Thus, using $|S|$ to denote the number of elements in $S$, we have.

$$
\sum\left|S_{i}\right|+\sum\left|S_{i j}\right|=2 k-5,
$$

since the valence of 0 is $2 k-2$.

We also define

$$
\widetilde{S_{i}}=\{x \mid x \text { is adjacent to } i \text {, but not to } 0\} .
$$

Note that $x \in \widetilde{S_{i}}$ implies $x$ is not adjacent to either $j$ or $k$, for if $x$ is adjacent to both $i$ and $j$, then

$$
H(0, x, i, j, k)=H_{1}(a, d, b, c, \alpha) .
$$

We define a clique to be a graph of which each pair of vertices is adjacent.

Lemma 5.1. If $S_{i} \neq \emptyset$, and $j \neq i$, then $\widetilde{S_{j}}$ is a clique.

Proof. Assume otherwise. Then there are two vertices, say 4 and 5 , in $\widetilde{S_{j}}$, which are not adjacent. Let 6 be any vertex in $S_{i}$. Since $\{0 ; 6, j, k\}$ is a claw, by a previous remark, neither 4 nor 5 is adjacent to 6 . It follows that $H\{0, i, j, k, 4,5,6\}=H_{2}\{a, b, c, f, d, e, \alpha\}$.

Lemma 5.2. If $S_{i j} \neq \emptyset$, and $k \neq i, j$, then $S_{k}=\emptyset$.

Proof. To fix ideas assume $k=3$, and let $4 \in S_{3}, 5 \in S_{12}$. Since there are at least three vertices (namely $1,3,4$ ) adjacent to 0 but not to 2 , there must be at least 3 vertices 6,7 , and 8 in $\widetilde{S_{2}}$. They form a clique, by Lemma 5.1. Either 5 is adjacent to at least two vertices in $\widetilde{S_{2}}$ (say 6 and 7), or 5 is not adjacent to at least two vertices in $\widetilde{S_{2}}$ (say $6^{\prime}$ and $7^{\prime}$ ). In the former case, the graph $H(0,1,2,3,4,5,6,7)=G_{3}(a, d, e, b, c, f, g, h)$. In the latter case, the graph $H\left(0,1,2,3,4,5,6^{\prime}, 7^{\prime}\right)=G_{4}(a, d, e, b, c, f, g, h)$.

Lemma 5.3. $\left|S_{i j}\right| \leqq 10$.

Proof. To fix ideas, assume $i=1, j=2$, and let $4 \in \widetilde{S_{2}}$. Now let $x, y$, $\in S_{12}$, and assume 4 is adjacent to neither $x$ nor $y$. Then $x$ must be adjacent to $y$, otherwise $H(1,2, x, y, 4)=H_{1}(a, d, b, c, \alpha)$. Next, assume 4 
is adjacent to both $x$ and $y$. Then $x$ must be adjacent to $y$, otherwise $H(1,2, x, y, 4)=H_{3}(a, b, c, d, \alpha)$. To summarize, the subset $S_{12}^{*}$ of $S_{12}$ consisting of vertices each of which is not adjacent to 4 must be a clique; the subset $S_{12}^{* *}$ consisting of vertices each of which is adjacent to 4 is also a clique. If $\left|S_{12}\right|>10$, then, since $S_{12}=S_{12}^{*} \cup S_{12}^{* *}$, either $\left|S_{12}^{*}\right| \geqq 6$ or $\left|S_{12}^{* *}\right|$ $\geqq 6$.

In the former case $H(4,1,2,5,6,7,8,9,10)=G_{5}(a, b, c, d, e, f, g, h, i)$ where $5,6,7,8,9$, and 10 are 6 vertices of $S_{12}^{*}$. In the latter case

$$
H(4,1,2,5,6,7,8,9,10)=G_{6}(a, b, c, d, e, f, g, h, i)
$$

where $5,6,7,8,9$ and 10 are 6 vertices of $S_{12}^{* *}$.

6. The nonexistence of claws in the edge regular case. The graph is called edge regular if every edge is contained in the same number of triangles. Since $H$ is edge regular, and each diagonal entry of $A^{3}$ is $2(k-1)(k-2)$ (from 4.7), it follows that every edge of $H$ is contained in exactly $k-2$ triangles. Assume a claw as in $\$ 4$.

Lemma 6.1. For each $i, \widetilde{S_{i}}$ contains two nonadjacent vertices.

Proof. To fix ideas, take $i=3$. Since the valence of 3 is $2 k-2, \widetilde{S_{3}}$ must contain $k-1$ vertices. We assume they form a clique, and will establish a contradiction. Because $\widetilde{S_{3}}$ is a clique, each edge joining 3 to a vertex in $\widetilde{S_{3}}$ is contained in $k-2$ triangles where the third vertex is in $\widetilde{S_{3}}$. Consequently, by the edge regularity, 0,3 , and all vertices adjacent to 0 and 3 form a clique. In turn, this implies that all vertices adjacent to 0 but not to 3 form a clique. But 1 and 2 are adjacent to 0 and not to 3 , yet 1 and 2 are not adjacent to each other.

Lemma 6.2. If $k \neq 4$, then $H$ contains no claw.

Proof. By Lemma 6.1, each $\widetilde{S_{i}}$ contains two vertices not adjacent to each other. By Lemma 5.1, this means each $S_{i}$ is empty. Using the edge regularity condition on the edges $(0,1)(0,2)$ and $(0,3)$, and adding, we have

$$
2 \sum\left|S_{i j}\right|=3(k-2)=3 k-6 .
$$

By (5.1),

$$
2 \sum\left|S_{i j}\right|=4 k-10 .
$$

Therefore, $k=4$.

Lemma 6.3. If $k=4$, then $H$ contains no claw.

Proof. Assume $k=4$, and we have the claw $0,1,2,3$. By edge regu- 
larity, $\left|S_{12}\right|=\left|S_{13}\right|=\left|S_{23}\right|=1$, and let $\{4\}=S_{12}, \quad\{5\}=S_{13},\{6\}=S_{23}$. We then have

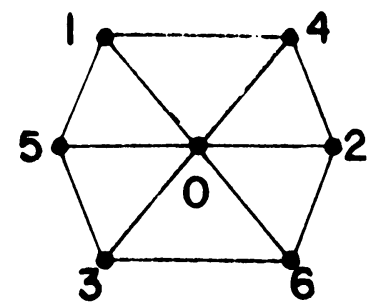

The valence of each vertex of $H$ is $2 k-2+6$. There must be a vertex (say 7) adjacent to both 1 and 4 , since $k-2=2$, and 7 cannot be adjacent to 0 . If 7 is adjacent to any vertex in $\{2,3,5,6\}$, it must be adjacent to at least one other, otherwise $H(1,2,3,4,5,6,7)$ would be $H_{4}$ or $H_{5}$.

Suppose 7 is adjacent to 5 . Then, in order to avoid both $H_{4}$ and $H_{6}, 7$ must be adjacent to 2 or 3 . Without loss of generality, we can take 7 adjacent to 2 and $5 . H\{0,2,5,7,1\}$ is $H_{3}(a, c, d, b, \alpha)$. Hence 7 cannot be adjacent to 5. In a like manner we can show that 7 is not adjacent to 2 . Suppose 7 is adjacent to neither 2 nor 5 , but is adjacent to 6 . Then $H\{4,6,0,7,2\}=H_{3}(a, b, d, c, \alpha)$. Hence 7 is not adjacent to 6. Similarly 7 cannot be adjacent to 3 .

Therefore, 7 is not adjacent to $2,3,5$, or 6 . In like manner we can find distinct vertices $8,9,10,11,12$ all distinct, with 8 adjacent to 2 and 4,9 adjacent to 2 and 6,10 adjacent to 6 and 3,11 adjacent to 3 and 5,12 adjacent to 5 and 1. Referring back to (2.1) and (2.2), we have

$$
\sum_{j=7}^{12} b_{0 j}\left(b_{0 j}-1\right)=6 \times 2 \cdot 1=12 \leqq 2(\lambda-1) \cdot 3 .
$$

Therefore, $\lambda-1 \geqq 2$ or $\lambda \geqq 3$. Since $k=4$, this means $\lambda=3$. $v=5, v k=20$. Now we shall use (2.3) and (2.4). If a vertex $j$ is not connected to 0 by a path of length 2 , then $c_{0 j}=-1$. This means that the number of vertices at distance greater than two from 0 must be at most one.

Now each of $\{1, \cdots, 6\}$ has valence 6 , and we have already identified, for each, 5 adjacent vertices. Therefore, there are at most 6 more vertices at distance two from 0 . If there were exactly 6 such vertices, we would have identified 18 vertices at distance one or two from 0 , and not yet have a violation of (2.4). But if there are fewer than 6 such vertices, we "would have a violation of (2.4).

Let 13 be adjacent to 4 , but not to 1 or 2 . If 13 were adjacent to 5 , then $\{13,4,0,5,1\}$ would form a graph $H_{3}$. Similarly, 13 cannot be adjacent to 6. If 13 were not adjacent to 3 , we would have $H\{1,2,3,4,5,6,13\}$ 
$=H_{7}(a, c, e, b, f, d, \alpha)$. Therefore, 13 must be adjacent to 3 , so we do not have six additional vertices at distance two from 0 , and hence have a violation of (2.4).

7. Proof that $H$ is edge regular if $k>3$. In this section, we assume $H$ is not edge regular and $k>3$, and show this leads to a contradiction.

If $H$ is not edge regular, then there is some edge (say $(0,1))$ contained in $k-3-a$ triangles $(a \geqq 0)$, and every edge of $H$ is contained in at least $k-3-a$ triangles.

Lemma 7.1. There exists a claw $\{0,1,2,3\}$.

Proof. If no such claw existed, then we would have $k+a$ vertices adjacent to 0 and not adjacent to 1 all forming a clique. For each such vertex $j,\left(A^{2}\right)_{0 j}$ would be at least $k+a-1$. For each other vertex $j$, adjacent to 0 , we have $\left(A^{2}\right)_{0 j}$ is at least $k-3-a$. Therefore, $\left(A^{3}\right)_{00}$ would be at least $(k-2-a)(k-3-a)+(k+a)(k+a-1)$, which exceeds $2(k-1)$ $\cdot(k-2)$.

Lemma 7.2. $S_{1}=S_{2}=S_{3}=\emptyset . \quad\left|S_{23}\right|=k-2+a$.

Proof. The same reasoning which established the claw proves that $\widetilde{S_{1}}$ is not a clique. Therefore, $S_{2}=S_{3}=\emptyset$, by Lemma 5.1. Since the number of vertices adjacent to 0 and 1 is $k-3-a$, the number of vertices adjacent to 0 but not adjacent to 1 is $k+a$, which means $\left|S_{23}\right|=k-2+a$. But $k-2+a>0$, which (by Lemma 5.2), implies $S_{1}=\emptyset$.

Lemma 7.3. If $k>3, H$ is edge regular.

Proof. If $H$ is not edge regular, the previous lemmas of this section apply, and we have a claw $\{0 ; 1,2,3\}$ with $\left|S_{23}\right|=k-2+a,\left|S_{12}\right|+\left|S_{13}\right|$ $=k-3-a$. Without loss of generality, we can assume

$$
\left|S_{12}\right| \geqq \frac{k-3-a}{2} .
$$

By Lemma $5.3,\left|S_{23}\right|=k-2+a \leqq 10$. Therefore, $k \leqq 12$.

Now let us make the tentative assumption that $\lambda<k-1$. By (1.2), this means that, in case $k=12$, for example, $\lambda \leqq 6$. Therefore, the right side of (2.2) is at most 110. But, in (2.2), the left side is at least $b_{23}\left(b_{23}-1\right)$ $+b_{21}\left(b_{21}-1\right)$, which is

$$
(11+a)(10+a)+\left[\frac{12-a}{2}\right]\left(\left[\frac{12-a}{2}\right]-1\right) .
$$

Since $a \geqq 0$, this is a contradiction. This line of reasoning eliminates all possible values of $k, 4 \leqq k \leqq 12$, with $\lambda<k-1$, except $k=9$.

If $k=9$, and $\lambda<k-1$, then $\lambda=6$ or $\lambda \leqq 4, \lambda \leqq 4$, the above reasoning 
applies. If $\lambda=6$, then, from (1.2), $v=13$. By (2.3) and (2.4), $c_{23}\left(c_{23}-1\right)$ $=\left|S_{23}\right| \cdot\left(\left|S_{23}\right|-1\right) \leqq 24$. But $\left|S_{23}\right| \geqq 7$, a contradiction.

Therefore, all we need consider is the case $\lambda=k-1$, so $v=k+1$. Since the right side of $(2.4)$ is then 2 , it follows that $\left|S_{23}\right| \leqq 2$. Since $k-2$ $\leqq\left|S_{23}\right|$, we have only to consider the case $k=4$. When $k=4,\left|S_{12}\right|=1$, $\left|S_{23}\right|=2$. Therefore, in $(2.4), c_{23}=2$. Since, in general $\left(A^{3}\right)_{00}=2(k-1)$ $(k-2)$, the number of edges in the graph subtended by vertices adjacent to 0 must be $(k-1)(k-2)$, or be 6 for the case $k=4$. But $\left|S_{12}\right|=1$, $' S_{23} \mid=2$ already picks out six edges, so that, if $S_{23}=\{4,5\}$, and $S_{12}=\{6\}$, we have, in the graph subtended by $\{2,3,4,5,6\}$ the graph $H_{1}$.

\section{The main theorem.}

Lemma 8.1. Let $H$ be edge regular and contain no claw. Then

(8.1) every edge of $H$ is contained in exactly one clique of order $k$,

(8.2) every maximal clique of $H$ contains $k$ vertices,

(8.3) every vertex is contained in two cliques of order $k$,

(8.4) there are $2 v$ cliques of order $k$.

Observe first that if 0,1 are adjacent vertices, then the $k-1$ vertices adjacent to 0 and not to 1 , together with 0 must form a clique of order $k$. Clearly every edge of $H$ is accounted for in a clique exactly once this way, which proves $(8.1),(8.2)$ and (8.3) are equally obvious. Let $T$ denote the total number of cliques of order $k$. Since every vertex is contained in two cliques, $k T=2 v k$, or $T=2 v$, which is (8.4).

THEOREM. Let $H$ be a regular connected graph on vk vertices, such that the distinct eigenvalues of its adjacency matrix $A=A(H)$ are

$$
2 k-2-2, k-2 \pm V^{\prime}(k-\lambda) \text {. }
$$

Then $H \cong L(I I(v, k, \lambda))$ unless $k=3, \lambda=2$, when there is exactly one exception.

Proof. If $k>3 H$ is edge regular and contains no claw and Lemma 8.1 applies. Let $\tilde{H}$ be the graph with $2 v$ vertices corresponding to the cliques of order $k$ in $H$, and two vertices of $\widetilde{H}$ adjacent if the corresponding cliques of $H$ have a common vertex. By Lemma $8.1, \widehat{H}$ is a regular connected graph on $2 v$ vertices, and $H$ is its line graph. The therorem will then follow from Lemma 4.2.

By Lemmas 6.2, 6.3 and 7.3 , the theorem holds if $k>3$. If $k=3$, and $\lambda=1, \mid 4]$ applies. If $k=3, \lambda=2$, and the theorem does not hold, $H$ is not edge regular, and $\lambda=2$. In this case $k-2=1$. Since $H$ is not edge regular, there exists an edge $(a, b)$ which is not contained in a triangle. Since the number of triangles containing a given vertex is $(k-1)(k-2)$ $=2$, we must have the following subgraph. 


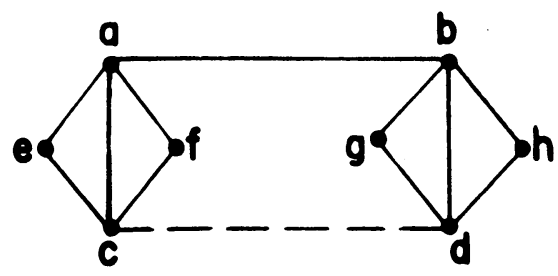

In case $k=3, \lambda=2, v=4, v k=12$, the polynomial of the graph is $\left(x^{3}-4 x\right) / 4$. Therefore there must be exactly eight paths of length 3 from $a$ to $b$. This, however, is impossible unless $c$ and $d$ are adjacent, so our subgraph becomes:

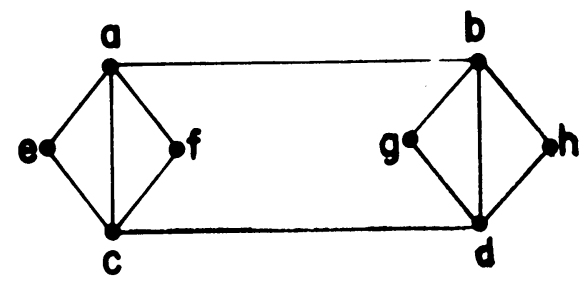

Now in order that $\left(A^{3}\right)_{f f}=4$, it is necessary that there exist vertices $i$ and $j$ such that $f, i, j$ form a triangle. Since the valence of every vertex is 4 , vertex $i$ cannot be adjacent to $a, c, b$, and $d$. Vertex $i$ must be adjacent to at least one of $e, g, h$ otherwise the vertices $a, b, c, e, f, g, h, i$ would subtend a graph $H_{8}$. But $i$ cannot be adjacent to $e$, otherwise $\{a, e, f, c, i\}$ would subtend $H_{3}$. Vertex $i$ cannot be adjacent to both $g$ and $h$, otherwise vertices $i, g, h, d, f$ will subtend $H_{1}$. So $i$ is adjacent to exactly one of $g$ and $h$, say $g$, and we have

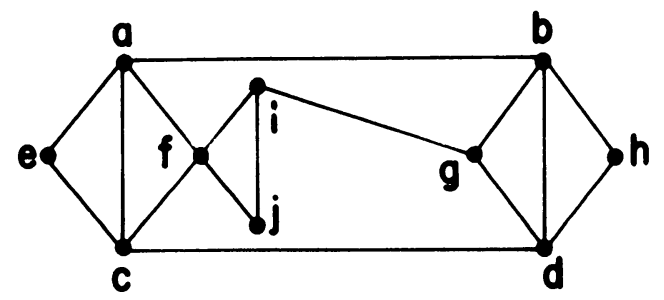

By the same argument, $j$ cannot be adjacent to $e$ and must be adjacent to one of $g$ and $h$. Now there are two possible cases. In the first case we assume $j$ to be adjacent to $h$. In the second case $j$ is adjacent to $g$. Let us consider the first case. In this case vertex $h$ cannot be adjacent to $e$, otherwise vertices $\{e, c, d, h, j\}$ subtend a graph $H_{1}$. Hence there is a new vertex $k$ adjacent to $h$ and to fulfill $A_{h h}^{4}=4, j$ and $k$ must be adjacent. In the subgraph $\{a, b, c, d, e, f, g, h, i, j, k\}$ valence of every vertex other than $i, g$ and $e$ is 4 . Vertices $i$ and $g$ are already adjacent. We have shown that vertices $i$ and $e$ cannot be adjacent. Hence the twelfth vertex $l$ is adjacent to $i$. It is easily checked that we get the following graph: 


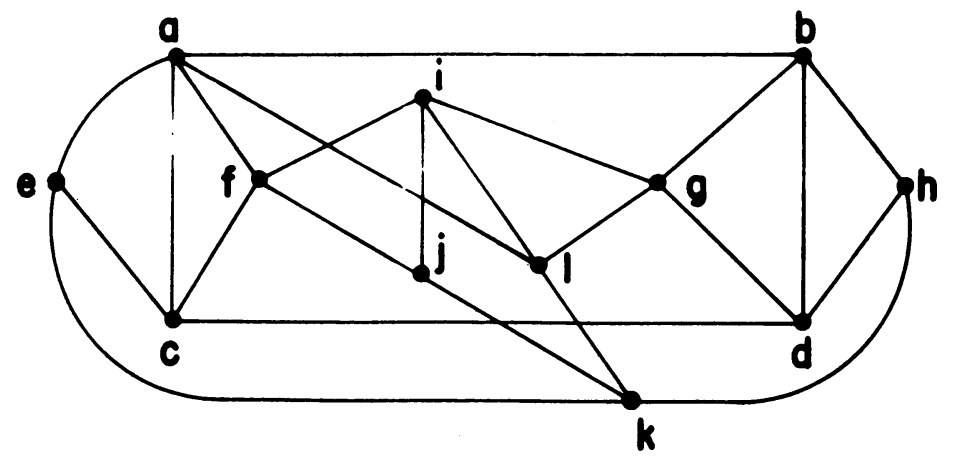

However for this graph $\sum_{r} b_{f r}\left(b_{f r}-1\right)=2$, where the summation is over all the vertices. This violates (2.2). Hence this graph does not satisfy the hypothesis.

Now let us consider the second case when $j$ is adjacent to $g$. In this case it is readily checked that we get the following graph

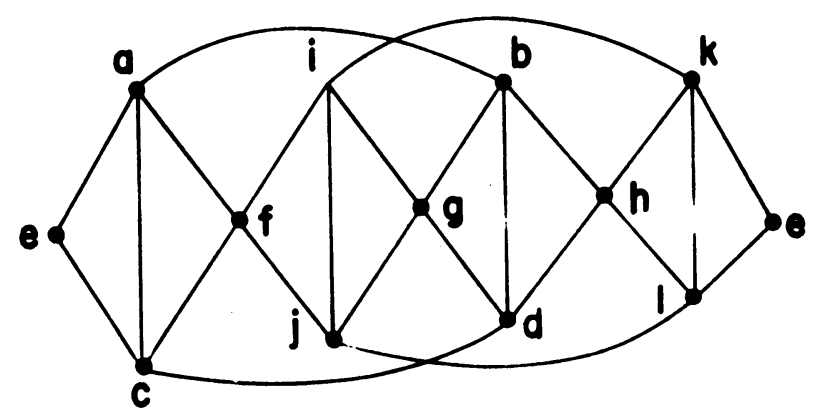

and this graph does satisfy the hypotheses.

\section{REFERENCES}

1. R. H. Bruck, Finite nets. II. Uniqueness and embedding, Pacific J. Math. 13 (1963), 421-457.

2. R. C. Bose, Strongly regular graphs, partial geometries and partially balanced designs, Pacific J. Math. 13 (1963), 389-419.

3. A. J. Hoffman, On the polynomial of a graph, Amer. Math. Monthly 70 (1963), 30-36.

4. $\ldots$, On the line graph of a projective plane, Proc. Amer. Math. Soc. 16 (1965), 297-302.

5. A. J. Hoffman and D. K. Ray-Chaudhuri, On the line graph of a finite affine plane, Canad. J. Math. (to appear).

InTERnational Business Machines Corporation,

YoRKTOWN HEIGHTS, NEW YORK 